\title{
DESFECHO DA APLICAÇÃO DA VENTILAÇÃO MECÂNICA NÃO INVASIVA NA BRONQUIOLITE
}

\author{
OUTCOME OF THE APPLICATION OF NONINVASIVE \\ MECHANICAL VENTILATION IN BRONCHIOLITIS
}

\author{
Gláucia Zuleide Stumm¹, Vagner Reinaldo Zingalli Bueno Pereira² \\ Jonas Michel Wolf ${ }^{3}$, Luiz Alberto Forgiarini Junior ${ }^{4}$
}

Clin Biomed Res. 2021;41(1):84-90

1 Centro Universitário

Metodista IPA. Porto Alegre,

Rio Grande do Sul, Brasil.

2 Universidade Luterana do Brasil (ULBRA). Canoas,

Rio Grande do Sul, Brasil.

3 Universidade Luterana do Brasil (ULBRA). Canoas, Rio Grande do Sul, Brasil.

4 Centro Universitário Metodista IPA. Porto Alegre, Rio Grande do Sul, Brasil.

Autor correspondente: Gláucia Z. Stumm glaucia.stumm@gmail.com.br Serviço de Fisioterapia, Hospital Geral Rua Prof. Antônio Vignoli, 255 95070-561, Caxias do Sul, RS, Brasil.

\section{RESUMO}

A bronquiolite é uma infecção viral que pode levar a insuficiência ventilatória, nestes casos a aplicação de ventilação não invasiva é uma opção ao tratamento convencional. O objetivo deste estudo é identificar o desfecho da aplicação de ventilação não invasiva em crianças com bronquiolite. Trata-se de uma revisão da literatura, com busca de artigos nas bases de dados Biblioteca Virtual em Saúde, Cochrane Library, PEDro, Pubmed, Scielo e Science Direct, sem restrição de período, a partir das palavras chave Noninvasive ventilation AND Bronchiolitis. De um total de 1.192 artigos encontrados, 11 foram inclusos no presente estudo, quatro abordaram o desfecho relacionado à aspectos clínicos gerais (aumento no uso de VNI; menor tempo de internação; redução da frequência respiratória e fração inspirada de oxigênio) e sete relataram o índice de sucesso ou falha, e apesar da diferença entre as médias (sucesso 88,5 versus falha 15,1) não houve diferença significativa. Conclui-se que o desfecho de falha tem alta prevalência (11,5\%), apesar disso, os estudos encontraram diferentes benefícios advindos do incremento na aplicação de VNI (redução da necessidade de ventilação mecânica invasiva, menor tempo de permanência em UTI, redução da mortalidade, melhora da frequência respiratória e da fração inspirada de oxigênio).

Palavras-chave: Ventilação não invasiva; Bronquiolite; Criança

\section{ABSTRACT}

Bronchiolitis is a viral infection that can lead to ventilatory failure. In such cases, the application of noninvasive ventilation (NIV) is an alternative to conventional treatment. The aim of this study is to identify the outcome of the application of NIV in children with bronchiolitis. This is a literature review whose search for articles included the Virtual Health Library, Cochrane Library, PEDro, PubMed, SciELO, and Science Direct databases, with no date restriction, based on the keywords Noninvasive ventilation AND Bronchiolitis. Of a total of 1192 articles found, 11 were included in the present study. Four addressed the outcome related to general clinical aspects (increased use of NIV, shorter hospital stay, and reduced respiratory rate and inspired oxygen fraction) and seven reported the success or failure rate. Despite the difference between the means (success 88.5 versus failure 15.1) there was no significant difference. In conclusion, the failure outcome has a high prevalence (11.5\%); however, studies have found different benefits arising from the increased application of NIV (reduced need for invasive mechanical ventilation, shorter intensive care unit stay, reduced mortality, and improved respiratory rate and inspired oxygen fraction).

Keywords: Noninvasive ventilation; Bronchiolitis; Child

\section{INTRODUÇÃO}

A bronquiolite é uma infecção viral, sendo o vírus sincicial respiratório o principal agente etiológico. Após a inoculação do vírus a criança permanece assintomática por 4 a 5 dias, passado este período os achados clínicos 
correspondem a infecção da via aérea superior (coriza, redução de apetite e irritabilidade). Posteriormente, ocorre a infecção de vias aéreas inferiores, que afeta principalmente os bronquíolos, gerando a instalação do quadro clínico comum da bronquiolite (taquipnéia, respiração superficial, aumento de esforço respiratório, febre, tosse produtiva, expiração prolongada, hiperinsuflação pulmonar, dispnéia e crises de sibilância) ${ }^{1-3}$.

Trata-se de uma das doenças mais comuns entre crianças em seus dois primeiros anos de vida, tem caráter sazonal, e apresenta a maior taxa mundial de hospitalização em crianças ${ }^{1}$. Estudos recentes tem demonstrado aumento na taxa de procura por atendimento e hospitalização devido a bronquiolite ${ }^{4}$.

A necessidade de hospitalização se deve ao agravamento dos sinais clínicos da bronquiolite, podendo evoluir para insuficiência respiratória, neste caso, é necessário instituir medidas que possibilitem o suporte ventilatório adequado para suprir as demandas do paciente, e a ventilação mecânica não invasiva (VNI) tem, cada vez mais, se tornado uma alternativa a intubação endotraqueal (IET), visto que esta última está associada ao maior número de complicações e maior permanência em unidade de terapia intensiva (UTI) ${ }^{5}$.

A VNI é a aplicação de pressão positiva nas vias aéreas do paciente por meio de interface não invasiva ${ }^{5}$ e vem sendo foco de estudo já há algum tempo, dessa forma, o objetivo desta revisão de literatura é identificar o desfecho da aplicação de $\mathrm{VNI}$ em crianças com bronquiolite.

\section{MATERIAIS E MÉTODO}

Este estudo consiste em uma revisão da literatura sobre o desfecho da aplicação de VNI na bronquiolite. Desta forma, foram determinadas as palavras chave de acordo com os Descritores em Ciências da Saúde - DeCS, sendo elas: Noninvasive ventilation; Bronchiolitis. O único operador booleano utilizado para a combinação dos termos da pesquisa foi "AND".

Foi realizada busca eletrônica de artigos indexados nas bases de dados BVS (Biblioteca Virtual em Saúde), Cochrane Library, PEDro, Pubmed, Scielo e Science Direct. Não houve restrição quanto ao período de publicação, portanto foram selecionados todos os artigos disponíveis até a data da busca, que encerrou-se em janeiro de 2019.

Adotou-se como critérios de inclusão artigos originais, publicados nos idiomas português ou inglês, selecionados por" pesquisa direta. Foram excluídos artigos duplicados, que não atendiam a proposta do estudo ou que não respondiam ao problema da pesquisa.

A partir da leitura dos títulos, alguns artigos foram selecionados para a leitura dos resumos (abstracts), e os estudos que contemplaram os critérios de inclusão tiveram seu conteúdo analisado na íntegra de forma cega e independente por dois avaliadores que, posteriormente, os sumarizaram em uma tabela para melhor visualização dos resultados. As discordâncias de análise dos artigos foram resolvidas por consenso pelos dois avaliadores.

Os dados foram compilados e analisados com software SPSS $®$ (v.23.0, Chicago, IL Statistical Package for the Social Sciences). Para verificar o tipo de distribuição dos dados contínuos, foi realizado o teste Kolmogorov-Smirnov com correção de Lilliefors. As variáveis contínuas com distribuições gaussianas normais foram analisadas através do teste $\mathrm{t}$ de student. Para as variáveis categóricas foram utilizados o teste do qui-quadrado e/ou exato de Fisher, conforme recomendado. Foram utilizadas medidas de efeitos como Risco Relativo (RR), com intervalos de $95 \%$ de confiança (IC 95\%). Todos os testes empregados foram bilaterais e os valores de $p$ inferiores a 0,05 foram considerados significativos.

\section{RESULTADOS}

De um total de 1.192 artigos encontrados nas bases de dados, 11 foram selecionados para inclusão final no presente estudo. A Figura 1 apresenta a estratégia de seleção utilizada na busca.

Considerando apenas os estudos selecionados, houve uma média de 2,2 artigos publicados por ano, com um desvio padrão de 0,83 , sendo que o mínimo foi de um artigo em 2008 e o máximo foram três nos anos de 2017 e 2018.

Os estudos foram realizados em sua maioria na Europa. Em relação ao delineamento, a maioria dos artigos inclusos (6) tratavam-se de estudos retrospectivos, apenas 3 tratavam-se de ensaios clínicos, 1 era caso controle e 1 descritivo prospectivo.

As pesquisas apresentaram grande variabilidade em relação ao tamanho da amostra incluindo de 16 a 7.111 indivíduos, sendo que a soma dos números amostrais das pesquisas totalizou 9.020 crianças, que eram em sua maioria do sexo masculino, com idade inferior a 1 ano de vida.

A sumarização dos estudos inclusos encontrase na Tabela 1. Um total de 11 artigos analisaram a aplicação da VNI na bronquiolite, no entanto quatro abordaram aspectos clínicos gerais e apenas sete relataram o índice de sucesso ou falha, respondendo a pergunta desta pesquisa.

Os sete artigos que abordaram o desfecho da VNI totalizaram um número amostral de 726 pacientes, sendo a média 104 (mínimo de 15 e máximo de 285). Na Figura 2 comparou-se a diferença de médias entre os pacientes que falharam versus os que obtiveram sucesso na VNI. Apesar das médias variarem (sucesso 88,5 versus falha 15,1 ) não foi observada diferença estatisticamente significativa $(p=0,08)$. 


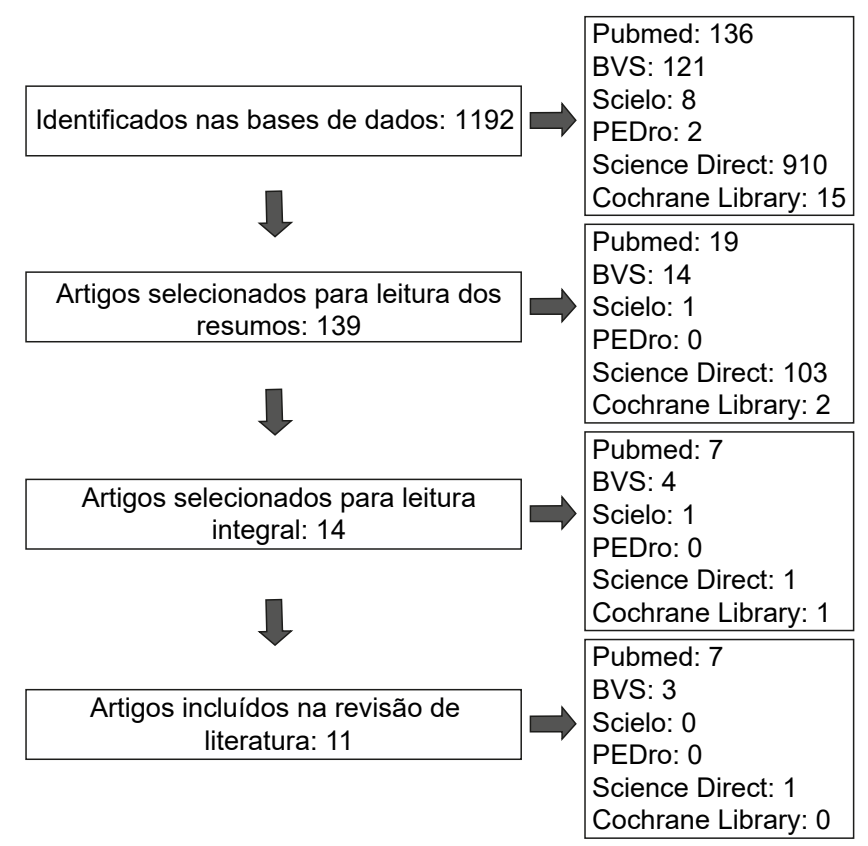

Figura 1: Estratégia de seleção dos artigos avaliados no presente estudo.

Tabela 1: Caracterização dos estudos incluídos na presente revisão

\begin{tabular}{|c|c|c|c|c|}
\hline Autor / Ano & Delineamento & $\mathbf{n}$ & Método & Principais resultados \\
\hline $\begin{array}{l}\text { Javouhey } \\
\text { et al. }{ }^{6} 2008\end{array}$ & $\begin{array}{l}\text { Estudo } \\
\text { retrospectivo }\end{array}$ & 80 & $\begin{array}{l}\text { Comparação de dois } \\
\text { períodos: } 2003 \text { a } 2004, \\
\text { onde utilizou-se apenas VM } \\
\text { (período VM) e } 2004 \text { a } 2005 \\
\text { onde a VNI foi o tratamento } \\
\text { de primeira escolha } \\
\text { (período VNI). }\end{array}$ & $\begin{array}{l}\text { No período VM foi registrado maior número de } \\
\text { meninos ( } p<0,05) \text { e de apnéias na admissão } \\
(p<0,01) \text {. Durante o período VNI houve redução } \\
\text { de VM }(p<0,001) \text { e uso de antibióticos }(p<0,01) \text {, } \\
\text { não foram registrados casos de PAV }(p<0,05) \text {. } \\
\text { No período VNI } 10 \text { crianças ( } 37 \%) \text { precisaram } \\
\text { oxigênio suplementar por mais } 8 \text { dias comparado } \\
\text { a } 33 \text { crianças }(65 \%) \text { no grupo VM }(p<0,05) \text {. } \\
\text { O tempo de permanência hospitalar e duração da } \\
\text { ventilação foram similares. Das } 15 \text { crianças que } \\
\text { utilizaram VNI, } 5 \text { falharam ( } 2 \text { co-infecção } \\
\text { por bactéria pulmonar, } 1 \text { infecção urinária, } \\
2 \text { prematuras cardíacas). }\end{array}$ \\
\hline $\begin{array}{l}\text { Ganu et al. }{ }^{7} \\
2012\end{array}$ & $\begin{array}{l}\text { Estudo } \\
\text { de coorte } \\
\text { retrospectivo }\end{array}$ & 520 & $\begin{array}{l}\text { Revisão da base de dados } \\
\text { de uma UTI pediátrica em } \\
\text { busca de pacientes admitidos } \\
\text { por bronquiolite no período } \\
\text { compreendido entre janeiro } \\
\text { de } 2000 \text { e dezembro de } 2009 \text {. }\end{array}$ & $\begin{array}{l}\text { De um total de } 285 \text { pacientes que utilizaram VNI, } \\
237(83,2 \%) \text { obtiveram sucesso, e } 48(16,8 \%) \\
\text { falharam. O tempo de permanência na UTI é } \\
\text { menor nos pacientes submetidos apenas à VNI } \\
(2,38+2,43 \text { dias) comparado aos pacientes em VM } \\
(5,19+6,34 \text { dias) ou aqueles que falharam a VNI } \\
\text { e necessitaram VM ( } 8,41+3,44 \text { dias). Na falha da } \\
\text { VNI há maior presença do vírus sincicial respiratório } \\
\text { ( } p=0,002) \text { e de comorbidade ( } p=0,009) \text {. O uso de } \\
\text { VNI aumentou }(2,8 \% \text { ao ano) com declínio de VM } \\
\text { ( } 1,9 \% \text { ao ano) ( } p=0,002) \text {. A taxa de falha de VNI } \\
\text { decaiu de } 31,8 \text { para } 13,5 \% \text { durante o estudo. }\end{array}$ \\
\hline $\begin{array}{l}\text { Soilly et al. }{ }^{8} \\
2012\end{array}$ & $\begin{array}{l}\text { Estudo } \\
\text { retrospectivo }\end{array}$ & 467 & $\begin{array}{l}\text { Descrição da população de } \\
\text { crianças admitidas em UTIs } \\
\text { por bronquiolite entre } 2005 \text { e } \\
2006 \text { na França e verificação } \\
\text { de concordância destes com } \\
\text { uma base de dados nacional. }\end{array}$ & $\begin{array}{l}\text { Menor idade gestacional é um fator de risco } \\
\text { independente para VM ( } p=0,0003) \text {. Presença } \\
\text { de vírus sincicial respiratório é fator de risco } \\
\text { independente para uso de CPAP }(p=0,0280) \text {. } \\
\text { Dados sobre o desfecho da VNI não foram } \\
\text { apresentados. }\end{array}$ \\
\hline
\end{tabular}


Tabela 1: Continuação

\begin{tabular}{|c|c|c|c|}
\hline Autor / Ano & Delineamento & $\mathbf{n}$ & Método \\
\hline $\begin{array}{l}\text { Wolfer } \\
\text { et al. }{ }^{9} 2015\end{array}$ & $\begin{array}{l}\text { Estudo } \\
\text { multicêntrico } \\
\text { de coorte } \\
\text { retrospectiva }\end{array}$ & 7.111 & $\begin{array}{l}\text { Evolução de aspectos da VNI } \\
\text { em crianças admitidas em } \\
\text { treze UTls Italianas durante } 5 \\
\text { anos (2006-2012). }\end{array}$ \\
\hline $\begin{array}{l}\text { Del Castillo } \\
\text { et al. }^{10} 2015\end{array}$ & $\begin{array}{l}\text { Estudo } \\
\text { observacional } \\
\text { retrospectivo }\end{array}$ & 196 & $\begin{array}{l}\text { Comparação da evolução } \\
\text { do uso de VNI e VM por } \\
\text { dois períodos iguais de seis } \\
\text { anos (2001 a } 2006 \text { e } 2007 \\
\text { a } 2012 \text { ) em pacientes com } \\
\text { bronquiolite. }\end{array}$ \\
\hline
\end{tabular}

\begin{tabular}{ll}
\hline Pedersen & Estudo \\
et al. ${ }^{11} 2017$ & retrospectivo
\end{tabular}

49 Comparação de CPAP e cânula nasal de alto fluxo para o tratamento da bronquiolite (2013 a 2015).

\begin{tabular}{llrl}
\hline Flores- & Estudo & 262 & $\begin{array}{l}\text { Descrição epidemiológica } \\
\text { González }\end{array}$ \\
prospectivo & e do manejo terapêtico \\
et al. ${ }^{12} 2017$ & multicêntrico pacientes internados \\
& & em UTIs da Espanha no \\
& & período compreendido entre \\
& & 2014 e 2015.
\end{tabular}

\begin{tabular}{|c|c|c|c|c|}
\hline & & & & \\
\hline $\begin{array}{l}\text { Milesı et al. } \\
2017\end{array}$ & $\begin{array}{l}\text { Ensaio clinico } \\
\text { randomizado } \\
\text { multicêntrico }\end{array}$ & 142 & $\begin{array}{l}\text { Comparaçao do uso de } \\
7 \mathrm{cmH} 2 \mathrm{O} \text { em CPAP com fluxo } \\
\text { de oxigênio à } 2 \mathrm{~L} / \mathrm{Kg} / \mathrm{min} \text { na } \\
\text { cânula nasal de alto fluxo } \\
\text { em crianças de } 6 \text { meses } \\
\text { de idade com bronquiolite } \\
\text { quanto ao desfecho de falha } \\
\text { em } 24 \mathrm{~h} \text {, taxa de sucesso, } \\
\text { tempo de permanência, entre } \\
\text { outros }(11 / 2014-03 / 2015) \text {. }\end{array}$ & $\begin{array}{l}\text { grupo CPAP e } 36 \text { de } 71 \text { crianças }(50,7 \%) \text { no } \\
\text { grupo cânula nasal de alto fluxo, diferença no } \\
\text { risco de falha } 19 \% \text { ( } 95 \% \text { Cl- } 35 \text { para } 3 \%) \text { não } \\
\text { permitiu concluir sobre a não inferioridade } \\
\text { da cânula nasal de alto fluxo. A análise de } \\
\text { superioridade demonstrou risco relativo de } \\
\text { sucesso } 1,63 \text { (95\% Cl } 1,02-2,63) \text { maior com } \\
\text { VNI. Os demais dados não apresentaram } \\
\text { diferença estatística entre os grupos. }\end{array}$ \\
\hline $\begin{array}{l}\text { Mecklin } \\
\text { et al. }{ }^{14} 2018\end{array}$ & $\begin{array}{l}\text { Estudo } \\
\text { caso-controle }\end{array}$ & 105 & $\begin{array}{l}\text { Avaliação da intervenção para } \\
\text { bronquiolite em UTI durante } \\
16 \text { anos (2000 a 2015). }\end{array}$ & $\begin{array}{l}\text { A VNI foi o suporte ventilatório de primeira } \\
\text { escolha em } 24(23 \%) \text { crianças, e } 5(21 \%) \\
\text { falharam necessitando VM. }\end{array}$ \\
\hline
\end{tabular}


Tabela 1: Continuação

\begin{tabular}{|c|c|c|c|c|}
\hline Autor / Ano & Delineamento & $\mathbf{n}$ & Método & Principais resultados \\
\hline $\begin{array}{l}\text { Mayordomo- } \\
\text { Colunga } \\
\text { et al. }{ }^{15} 2018\end{array}$ & $\begin{array}{l}\text { Estudo } \\
\text { prospectivo, } \\
\text { randomizado } \\
\text { cruzado. }\end{array}$ & 16 & $\begin{array}{l}\text { Mensuração em } 30,60,90 \\
\text { e } 120 \text { min da escala clínica } \\
\text { modificada para pontuação de } \\
\text { asma, FC, FR, FiO2 e SpO2, }\end{array}$ & $\begin{array}{l}\text { A escala clínica modificada para pontuação de } \\
\text { asma no grupo capacete Helmet foi de } 4,8+1 \\
\text { para } 3+0,6 \text { aos } 60 \text { min }(p<0,001) \text { e para } 2,7+ \\
1,7 \text { aos } 120 \text { min }(p<0,001) \text {, já no grupo pronga } \\
\text { nasal o decréscimo foi de } 4,2+0,9 \text { para } 2,8+0,9 \\
\text { aos } 60 \text { min }(p<0,001) \text { e para } 2,9+0,9 \text { aos } 120 \\
\text { min }(p<0,001) \text {. A frequência cardíaca diminuiu no } \\
\text { grupo pronga nasal em } 30 \text { min. }\end{array}$ \\
\hline
\end{tabular}

e uso de sedação.

Comparação da eficácia da oferta de VNI por pronga nasal a capacete de Helmet.
A frequência respiratória apresentou redução significativa aos 90 min no grupo capacete de Helmet $(p<0,033)$, enquanto que no grupo pronga nasal isso só ocorreu depois de 6 horas $(p<0,01)$. Não foram encontrados valores significativos para a avaliação de uso de sedação, FiO2 e $\mathrm{SpO} 2$. Ao comparar a eficácia das técnicas não foram encontrados valores significativos para as variáveis avaliadas. Ocorreram 6 falhas de VNI, sendo 3 em cada grupo.

\begin{tabular}{|c|c|c|c|}
\hline $\begin{array}{l}\text { Lal et al. }{ }^{16} \\
2018\end{array}$ & $\begin{array}{l}\text { Ensaio clínico } \\
\text { controlado e } \\
\text { randomizado }\end{array}$ & 72 & $\begin{array}{l}\text { Comparação da FR, score } \\
\text { de gravidade modificado } \\
\text { da Sociedade de Pediatria } \\
\text { da Nova Zelândia e Escore } \\
\text { de Silverman-Anderson } \\
\text { entre dois grupos (CPAP } \\
\text { x tratamento padrão) após } \\
\text { uma hora de tratamento en } \\
\text { pacientes com bronquiolite } \\
(11 / 2014 \text { a 03/2016). }\end{array}$ \\
\hline
\end{tabular}

\begin{abstract}
No grupo CPAP 14 de 32 paciente apresentaram melhora significativa da FR (decréscimo) comparado a 5 de 35 pacientes do grupo tratamento padrão $(p=0,008)$. As médias (DP) da FR $[8,0(5,8)$ vs $5,1(4,0)$, $p=0,02]$, Escore de Silverman-Anderson $[0,78$ $(0,87)$ vs $0,39(0,73), p=0,029]$ e Escore de gravidade modificado da Sociedade de Pediatria da Nova Zelândia $[2,5(3,02)$ vs $1,08(1,3), p=0,012]$ foram significativamente diferentes entre o grupo CPAP e tratamento padrão consecutivamente.
\end{abstract}

VM: Ventilação mecânica invasiva; VNI: Ventilação mecânica não invasiva; UTI: Unidade de terapia intensiva; CPAP: Pressão positiva contínua nas vias aéreas; FC: Frequência cardíaca; FiO2: Fração inspirada de oxigênio; FR: Frequência respiratória; PAV: Pneumonia associada a ventilação; SpO2: Saturação periférica de oxigênio.

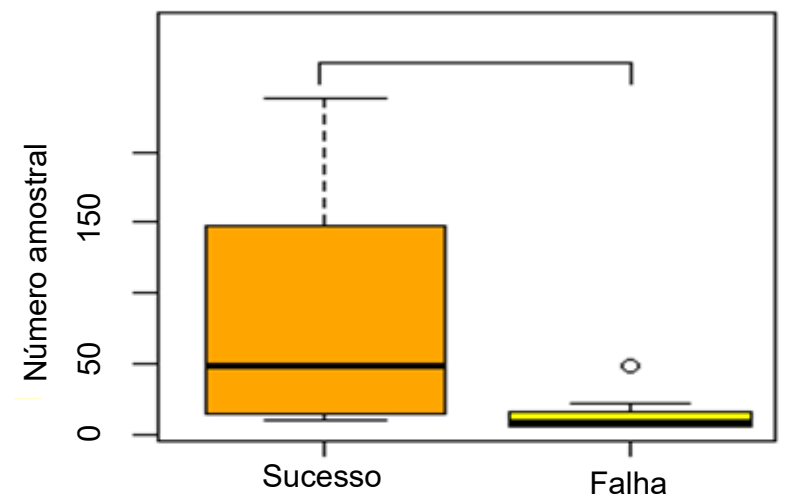

Figura 2: Representação gráfica dos dados de sucesso versus falhas na VNI oriundos dos estudos revisados.

Comparou-se o estudo Ganu et al. ${ }^{7}$ com os demais de forma isolada, visto que foi este o trabalho que apresentou maior número de falha, desta forma, objetivou-se verificar em quantas vezes era maior 0 riscos de falha nos pacientes do estudo de Ganu et al. ${ }^{7}$. Estes dados são apresentados na Tabela 2.

Nos artigos avaliados, a prevalência geral de falha na VNI foi de $11,5 \%$. O artigo de Ganu et al. ${ }^{7}$ 
apresentou valor significativo para a associação de falha na VNI quando comparado à: Milési et al. ${ }^{13}$ $(p<0,01)$; Flores-González et al. ${ }^{12}(p<0,001)$; Wolfer et al. ${ }^{9}(p=0,02)$ e Mayordomo-Colunga et al. ${ }^{15}(p<0,01)$. Sendo que apresentou três vezes mais chances de falha na VNI em relação à Flores-González et al. ${ }^{12}$

Tabela 2: Comparação do risco de falha entre o estudo de Ganu et al.7e os demais artigos de forma isolada.

\begin{tabular}{|c|c|c|c|c|c|c|c|c|}
\hline \multirow{2}{*}{ Estudos } & \multirow{2}{*}{ Ano } & \multirow{2}{*}{ n total } & \multicolumn{2}{|c|}{ Falha VNI } & \multicolumn{2}{|c|}{ Sucesso VNI } & \multirow{2}{*}{ RR (IC95\%) } & \multirow{2}{*}{ Valor de $p^{*}$} \\
\hline & & & $\mathbf{n}$ & $\%$ & $\mathbf{n}$ & $\%$ & & \\
\hline Ganu et al. ${ }^{7}$ & 2012 & 285 & 48 & 17 & 237 & 83 & $0,54(0,35-0,83)$ & $<0,01$ \\
\hline Milési et al. ${ }^{13}$ & 2017 & 71 & 22 & 31 & 49 & 69 & 1,00 ref. $* *$ & \\
\hline Ganu et al. ${ }^{7}$ & 2012 & 285 & 48 & 17 & 237 & 83 & $3,03(1,61-5,68)$ & $<0,01$ \\
\hline Flores-González et al. ${ }^{12}$ & 2017 & 198 & 11 & 5,5 & 187 & 94,5 & 1,00 ref. $^{* *}$ & \\
\hline Ganu et al. ${ }^{7}$ & 2012 & 285 & 48 & 17 & 237 & 83 & $2,18(1,11-4,31)$ & 0,02 \\
\hline Wolfer et al. ${ }^{9}$ & 2015 & 117 & 9 & 10,5 & 108 & 89,5 & 1,00 ref. $^{* *}$ & \\
\hline Ganu et al. ${ }^{7}$ & 2012 & 285 & 48 & 17 & 237 & 83 & $0,50(0,23-1,08)$ & $0,07^{* * *}$ \\
\hline Javouhey et al. ${ }^{6}$ & 2008 & 15 & 5 & 33 & 10 & 77 & 1,00 ref. $^{* *}$ & \\
\hline Ganu et al. ${ }^{7}$ & 2012 & 285 & 48 & 17 & 237 & 83 & $0,80(0,35-1,83)$ & $0,61^{* * *}$ \\
\hline Mecklin et al. ${ }^{14}$ & 2018 & 24 & 5 & 21 & 19 & 79 & 1,00 ref. $^{* *}$ & \\
\hline Ganu et al. ${ }^{7}$ & 2012 & 285 & 48 & 17 & 237 & 83 & $0,23(0,16-0,33)$ & $<0,01$ \\
\hline Mayordomo-Colunga et al. ${ }^{15}$ & 2018 & 16 & 6 & 37,5 & 10 & 62,5 & 1,00 ref. $^{* *}$ & \\
\hline
\end{tabular}

RR: Risco Relativo; *Em negrito resultados estatisticamente significativos; ${ }^{* *}$ Ref. categoria de referência; ${ }^{* * *}$ Teste exato de Fisher.

\section{DISCUSSÃO}

Incluíram-se, nesta revisão, 11 artigos oriundos de diversas partes do mundo e publicados em distintos periódicos. Os estudos são apresentados com diferentes delineamentos de pesquisa e importantes resultados quanto aos desfechos da VNI na bronquiolite.

A redução da necessidade de ventilação mecânica invasiva (VM) foi descrita por dois estudos ${ }^{6,10}$ o que vem ao encontro do aumento na utilização da VNI relatada por Ganu et al. ${ }^{7}$ e também por Del Castillo et al. ${ }^{10}$, este último ainda afirma que houve menor mortalidade em pacientes submetidos apenas a $\mathrm{VNI}$ em relação aos que necessitaram $\mathrm{VM}^{10}$. Além destes, Florez-Gonzales et al. ${ }^{11}$ ao apresentar dados sobre pacientes internados em UTIs espanholas, aponta o uso de VNI como o segundo manejo terapêutico mais comum, ficando atrás apenas da oxigenioterapia.

O estudo de Javouhey et al. ${ }^{6}$ comparou um período onde os pacientes foram submetidos a VM, e um segundo período onde a $\mathrm{VNI}$ foi o tratamento de primeira escolha, não encontrou diferença significativa em relação ao tempo de permanência hospitalar, o que discorda de outros estudos em que os pacientes submetidos a VNI tiveram redução significativa no tempo de permanência em UTI ${ }^{7,10,12}$. A discordância entre os resultados pode estar relacionada com a diferença no período em que os estudos foram realizados.

A frequência respiratória $(F R)$ foi avaliada em estudos que buscavam comparar a eficácia em dois tratamentos distintos. Ao comparar a aplicação de VNI no modo CPAP à cânula nasal de alto fluxo ${ }^{11}$, o primeiro obteve resposta significativa de forma mais eficaz, corroborando aos achados de Lal et al. ${ }^{16}$ que comparou o uso de CPAP ao tratamento padrão. Já Mayordomo-Colungs et al. ${ }^{15}$ aplicou VNI por duas interfaces diferentes, a pronga nasal e o capacete de Helmet, e demonstrou que no segundo grupo as respostas significativas ocorrem de forma mais rápida.

Os parâmetros ventilatórios não foram apresentados na maioria dos estudos, porém a maior limitação deste estudo deve-se ao fato de haver grande variabilidade em relação aos objetivos dos artigos selecionados, entre eles, a comparação de diferentes técnicas e a descrição dos tratamentos empregados. Não foram encontrados estudos comparando modos ventilatório (CPAP x BIPAP).

O principal desfecho da VNI (sucesso ou falha), foi avaliado por sete dos onze artigos selecionados, e a prevalência geral de falha foi de $11,5 \%$, o que é considerada alta prevalência segundo a Organização Mundial da Saúde.

Apesar do aumento de produções científicas demonstrando a eficácia da VNI, a lacuna frente como melhor utilizar esta técnica em crianças com bronquiolite permanece. Sugerem-se novos estudos comparando diferentes modos ventilatórios e interfaces a fim de reduzir a prevalência do desfecho de falha na VNI.

Conclui-se que o desfecho de falha na aplicação de ventilação não invasiva em crianças com bronquiolite ainda tem alta prevalência $(11,5 \%)$, no entanto, os estudos encontraram diferentes benefícios advindos do incremento em sua aplicação (redução da necessidade de ventilação mecânica invasiva, menor tempo de permanência em UTI, redução da mortalidade, melhora da frequência respiratória e da fração inspirada de oxigênio), justificando a continuidade do seu uso em crianças com bronquiolite. 


\section{REFERÊNCIAS}

1. Midulla F, Nenna R. Bronchiolitis in infants and wheeze in preschool children. Breathe. 2011;8:32-7.

2. American Academy of Pediatrics Subcommittee on Diagnosis and Management of Bronchiolitis. Diagnosis and management of bronchiolitis. Pediatrics. 2006;118(4):1774-93.

3. Sarmento GJV, Carvalho FA, Peixe AAF. Fisioterapia respiratória em pediatria e neonatologia. 2 ed. São Paulo: Manole; 2011.

4. Rivera-Sepulveda A, Garcia-Rivera EJ. Epidemiology of bronchiolitis: a description of emergency department visits and hospitalizations in Puerto Rico, 2010-2014. Trop Med Health. 2017;45:24.

5. Yaman A, Kendirli T, Ödek Ç, Ateş C, Taşyapar N, Güneş M, et al. Efficacy of noninvasive mechanical ventilation in prevention of intubation and reintubation in the pediatric intensive care unit. J Crit Care. 2016;32:175-81.

6. Javouhey E, Barats A, Richard N, Stamm D, Floret D. Non-invasive ventilation as primary ventilatory support for infants with severe bronchiolitis. Intensive Care Med. 2008;34(9):1608-14.
7. Ganu SS, Gautam A, Wilkins B, Egan $J$. Increase in use of non-invasive ventilation for infants with severe bronchiolitis is associated with decline in intubation rates over a decade. Intensive Care Med. 2012;38(7):1177-83.

8. Soilly AL, Ferdynus $C$, Desplanches O, Grimaldi M, Gouyon JB. Paediatric intensive care admissions for respiratory syncytial virus bronchiolitis in France: results of a retrospective survey and evaluation of the validity of a medical information system programme. Epidemiol Infect. 2012;140(4):608-16.

9. Wolfler $A$, Calderini $E$, lannella $E$, Conti G, Biban P, Dolcini A, et al. Network of pediatric intensive care unit study group - evolution of noninvasive mechanical ventilation use: a cohort study among italian PICUs. Pediatr Crit Care Med. 2015;16(5):418-27.

10. Castillo BT, Lafever SNF, Sanguos CL, Sánchez LDC, Silva MS, Cid JL-H. Evolution of non-invasive ventilation in acute bronchiolitis. An Pediatr (Barc). 2015;83(2):117-22.

11. Pedersen MB, Vahlkvist $S$. Comparison of CPAP and HFNC in Management of Bronchiolitis in Infants and Young Children. Children (Basel). 2017;4(4):28.
12. Flores-González JC, MayordomoColunga J, Jordan I, Miras-Veiga A, Montero- Valladares $\mathrm{C}$, OlmedillaJodar M, et al. Prospective multicentre study on the epidemiology and current therapeutic management of severe bronchiolitis in Spain. Biomed Res Int 2017;2017:2565397.

13. Milési C, Essouri S, Pouyau R, Liet JM, Afanetti M, Portefaix A, et al. High flow nasal cannula (HFNC) versus nasal continuous positive airway pressure (nCPAP) for the initial respiratory management of acute viral bronchiolitis in young infants: a multicenter randomized controlled trial (Tramontane study). Intensive Care Med. 2017;43(2):209-16.

14. Mecklin M, Heikkilä $P$, Korppi M. The change in management of bronchiolitis in the intensive care unit between 2000 and 2015. Eur J Pediatr. 2018;177(7):1131-7.

15. Mayordomo-Colunga J, Rey C, Medina A, Martínez-Camblor P Vivanco-Allende A, Concha A. Helmet versus nasal-prong CPAP in infants with acute bronchiolitis. Respir Care. 2018;63(4):455-63.

16. Lal SN, Kaur J, Anthwal P, Goyal K, Bahl P, Puliyel JM. Nasal continuous positive airway pressure in bronchiolitis: a randomized controlled trial. Indian Pediatr. 2018;55(1):27-30.

Recebido: 5 set, 2020 Aceito: 29 dez, 2020 